\title{
Sophisticated Multi-phase Multi-flow Modeling of the Blast Furnace
}

\author{
Sami Alex ZAÏMI, Tomohiro AKIYAMA, ${ }^{1)}$ Jean-Bernard GUILLOT ${ }^{21}$ and Jun-Ichiro YAG ${ }^{3)}$ \\ Département Fonte \& Acier IRSID, BP 30320, 57283 Maizières-lès-Metz (CEDEX), France. \\ 1) Miyagi National College of Technology, Natori, Miyagi Prefecture, 981-1239 Japan. \\ 2) Ecole Centrale Paris, Grande Voie des Vignes, 92295 Chatenay Malabry, Fuance. \\ 3) Institute for Advanced Materials Processing, Tohoku University, Katahira, Aoba-ku, Sendai, 980-8577 Japan.
}

(Received on July 28, 1999; accepted in final form on November 29, 1999)

\begin{abstract}
This article introduces an improvement of an existing blast furnace total model called the Four Fluid Flow Model, maintained by the last author. The derivation of solid phase motion in the former model is now replaced by an external numerical code, which implements a very specific granular flow theory called hypoplasticity. The calculation method in the external solid flow model is based on the finite element method (FEM), and differs from the method used in the fluid flow model (finite volume method or FVM), hence their separation. Both models are run one after the other by exchanging data such as solid velocity field, drag forces and solid voidage, until convergence. One major issue of the additional solid flow model is its ability to calculate the shape of the dead man (the name of the stagnant zone inside the blast furnace), whereas its shape was prescribed in the fluid model. The solid flow model also introduces stress state dependence on voidage, and takes into account source and sink terms related to solid phase physical and chemical transformations.
\end{abstract}

KEY WORDS: blast furnace; finite element method; hypo-plasticity theory; metallurgical reactor.

\section{Introduction}

The blast furnace was treated as a black box, where different phases are continuously input, undergo physical and chemical transformations, and then flow out. It is regarded as one of the most complex counter-current metallurgical reactors in the field of chemical engineering, due to the high number of species interacting and physical or chemical phenomena involved.

Blast furnace operators now have a relatively good understanding of the internal mechanisms, thanks to intensive instrumentation or observation, temperature probes, gas analysis, pig iron and slag analysis, burden samples through tuyeres, quenching, etc. ${ }^{1)}$

It is now very tempting to construct purely numerical models, in order to predict the effects of parameter changes more accurately. Actual research efforts in blast furnace technology, performed by major steel companies all over the world, for example in Japan, Australia or Europe, aim to gather data for a broad range of working conditions into total models. This task is today made possible by recent improvements in computer science and numerical methods. ${ }^{2)}$

In the last twenty years, many studies attempting to describe all inner phenomena have been undertaken in several laboratories in Japan. These studies have been mainly supported by the Japanese steel companies, like Nippon Steel $^{3,4)}$ and Sumitomo Metals, ${ }^{5-8)}$ with contributions from many university research laboratories, among these the
Institute for Advanced Materials Processing in Tohoku University, ${ }^{9-11)}$ working under the frame of the Four Fluid Flow project sponsored by the Iron and Steel Institute of Japan.

The aim of the researchers was to build a total model describing all flows inside the blast furnace, and the interactions between them, including chemical reactions and heat transfer. Each flow or phenomenon is described by a specific sub-model, e.g. for burden charging, solid motion, liquid motion, gas motion, raceway dynamic, cohesive zone state, shaft and hearth conditions.

More significantly, gas and liquid flows, as well as chemical reactions and heat transfer, depend mainly on solid flow behavior. For instance, knowledge of the burden (solid phase) velocity field and stress field enables the distribution of voidage to be described, which makes it possible to properly compute the gas paths, which is mandatory to have a correct knowledge of reaction locations and heat transfer efficiency.

Besides, the connection between the dead-man profile, inner and unknown boundary of the blast furnace, and the wall profile, outer but known boundary of the blast furnace, could be studied with the help of reasonable models giving both velocity field and stress field.

However, unfortunately, solid flow theory has never been well understood and a survey of the different studies carried out so far in Japan shows that different approaches were taken to at least understand solid flow mechanisms. ${ }^{12)}$ All 
these attempted approaches have known advantages, but also known shortcomings.

In the Four Fluid Flow model, for instance, the solid phase was assumed to behave like a viscous fluid, characterized by a purely numerical constant viscosity, which was adjusted in the past by trial and error to validate small blast furnace experiments containing sand. In this model, the dead man shape was also prescribed before simulation, and was assumed to be an impassable boundary for solid flow. The curve that describes the dead man's limit is only a mere quadratic equation of the form

$$
Y=a X^{4}+b X^{2}+c
$$

where the coefficients $a, b, c$ are fixed arbitrary parameters. Note that $c$ is the imposed dead man's height on central axis. This formula was never validated, and surely does not take into account chemical reactions occurring inside the dead man, nor fluid flows crossing it.

In the present article, a new approach is described, in which the methodology of the combination of two different codes was examined. Indeed, solid flow sub-modeling is now made by a specific external numerical code, based on granular plasticity theory, and is used in connection with the already accomplished multi-fluid flow model that describes all other phenomena.

With this approach, the solid momentum equations are solved by a more accurate numerical model, which, in particular, does not prescribe a dead man shape before calculation, and is based on real experimental constants that better describe granular material behavior in all conditions, from rest to steady flow.

The coupling concerns interaction terms that appear in the solid's momentum equations, like drag forces with other phases, and material source-sink terms like coke combustion, coke gasification and ore fusion. In return, the solid flow model furnishes a solid velocity field, and a more accurate definition of solid void fraction that takes into account solid pressure level. The results confirm that this is a new sophisticated blast furnace simulation model with high accuracy.

\section{Multi-phase Flow Theories in the Blast Furnace}

\subsection{Finite Volume Approach of Fluid Flows}

The Four Fluid Flow model is a steady state, axisymmetric model that simulates the highly coupled mechanisms of four reacting phases (gas, liquid, lump solids, powders). The calculation domain ranges from slag surface up to burden surface. Each phase is described by its density, volumetric fraction, temperature, velocity field, enthalpy. Within each phase, each species is described by its mass or volume fraction.

The species are:

Gas: the carbon system $\left(\mathrm{CO} / \mathrm{CO}_{2}\right)$, the hydrogen system $\left(\mathrm{H}_{2} / \mathrm{H}_{2} \mathrm{O}\right)$, oxygen $\mathrm{O}_{2}$ (it also appears in the two previous systems), nitrogen $\mathrm{N}_{2}$ (not reactive), and $\mathrm{SiO}$.

Solid: iron oxides (hematite $\mathrm{Fe}_{2} \mathrm{O}_{3}$, magnetite $\mathrm{Fe}_{3} \mathrm{O}_{4}$, wustite $\mathrm{Fe}_{\mathrm{w}} \mathrm{O}$ ) and sometimes iron, moisture, guangue (composed by $\mathrm{SiO}_{2}, \mathrm{Al}_{2} \mathrm{O}_{3}, \mathrm{CaO}, \mathrm{MgO}$ ), $\mathrm{C}$ (as coke and dust). Note that $\mathrm{SiO}_{2}$ also appears inside dust.
Table 1. Transport equation coefficients.

\begin{tabular}{cccc}
\hline Transport equation & Field $\Phi$ & Diffusive coefficient $\mu$ & Source term $S$ \\
\hline continuity & $\begin{array}{c}\text { volume fraction } \\
\text { of species or phases }\end{array}$ & not relevant & chemical reaction rate \\
or phase transformation \\
momentum & $\begin{array}{c}\text { phase velocity } \\
\text { components }\end{array}$ & viscosity & $\begin{array}{c}\text { pressure gradients, } \\
\text { drag forces, gravity }\end{array}$ \\
energy & $\begin{array}{c}\text { phase temperature } \\
\text { or phase enthalpy }\end{array}$ & thermal diffusivity & $\begin{array}{c}\text { interphase heat } \\
\text { transfer and heat of } \\
\text { chemical reaction }\end{array}$ \\
\hline
\end{tabular}

Liquid: molten iron, also called pig iron (that includes solved [C] and [Si]), and slag (including solved [FeO] and $\left.\left[\mathrm{SiO}_{2}\right]\right)$.

Powder: ash, volatiles, carbon. Components are $\mathrm{C}, \mathrm{H}, \mathrm{O}$, $\mathrm{N}$.

The interaction terms between phases are of two types:

Mechanical: these are drag forces between one phase and another. In the case of gas-solid interaction, there is a perpendicular and a tangential component due to the inclined structure of layers.

Thermal: these are convective heat transfer between one phase and another, especially between gas and solid.

The chemical and physical phenomena considered are:

1. indirect and direct iron reduction

2. carbon oxidation (combustion, solution loss $\mathrm{C}+\mathrm{CO}_{2} \rightleftharpoons$ $2 \mathrm{CO}$, water gas reaction $\mathrm{C}+\mathrm{H}_{2} \mathrm{O} \rightleftharpoons \mathrm{CO}+\mathrm{H}_{2}$ )

3. silicon transfer

4. phase transformations (like softening and melting in the cohesive zone)

The formulation leads to the same general transport equation used to describe all flows, physical and chemical exchanges and species transport:

$$
\vec{\nabla} \cdot(\underbrace{\rho \vec{v} \Phi}_{\text {conv. }}-\underbrace{\mu \vec{\nabla} \Phi}_{\text {diff. }})=S
$$

Equation (2) represents the conservation of fluxes inside any control volume. The left hand side is decomposed into a convective part and a diffusive part. Table 1 summarizes the different terms for each phenomenon modelized.

The diffusion parameter $\mu$ is equal to the viscosity in the case of the momentum equation, or is equal to thermal conductivity in the case of the heat conservation equation. In the former case, the term $\Phi$ represents each phase velocity component in both the convective part and the diffusive part. In the latter case, the term $\Phi$ represents phase enthalpy in the convective part and phase temperature in the diffusive part.

In the species mass conservation equation, the source term is related to chemical reactions and phase transformations. More precisely, the source term for each species within each phase is the rate at which it appears or disappears. In the phase continuity equation, the source term for each phase is the sum of all the source and sink terms of its individual components.

In the enthalpy conservation equations, the source terms are interphase heat transfers, reaction heat and sensible heat, linked with chemical reactions and phase transformation respectively. In the fluid model, all phases (including the solid phase) are assumed to behave like a viscous fluid, described by the Navier-Stokes constitutive equation. ${ }^{13)}$ 


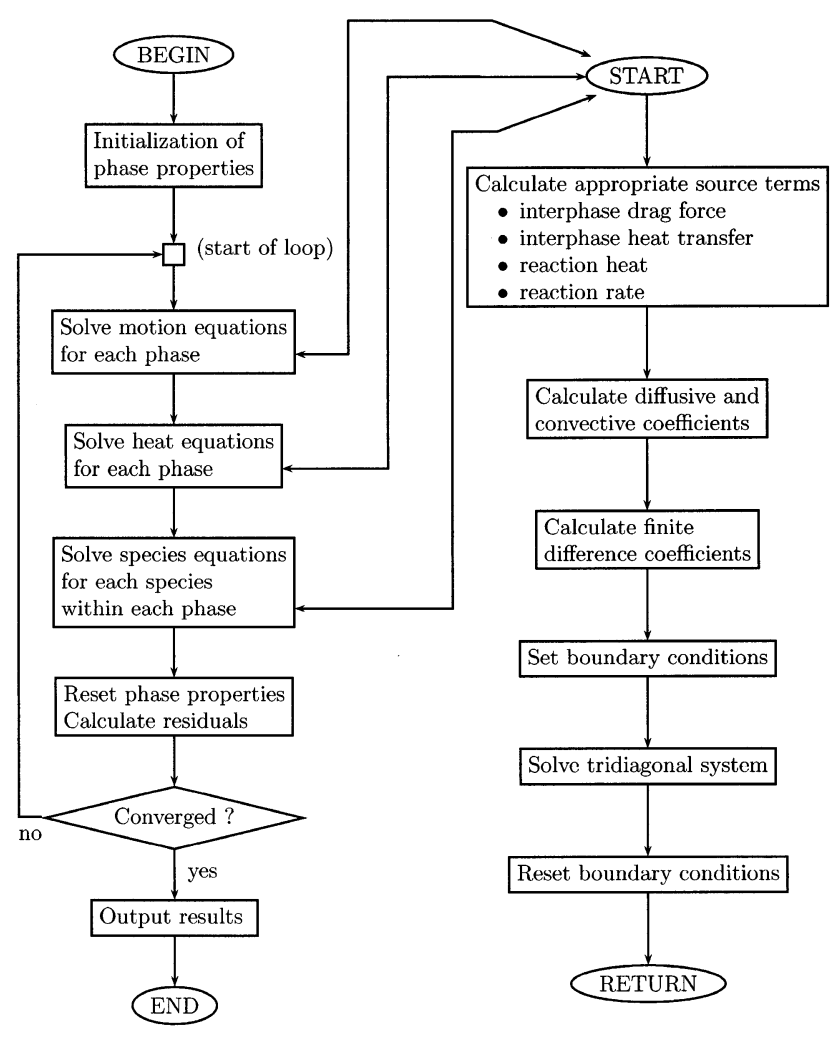

Fig. 1. Four fluid flow model's algorithm.

The parameters are dynamic viscosity $\mu$ and material density $\rho$. The source-like term at the right hand side contains the factors responsible for the motion. They are pressure gradients for continuous phases (solid, gas), gravity for discontinuous phases (liquids, fines), and interphase drag forces.

The major advantage of such a formulation is the possibility to solve all fields involved in the model with the same solver, ${ }^{14)}$ based in this case on the finite volume method (a technique where partial derivatives are replaced by finite differences). Like most numerical methods, several iterations are needed before reaching the solution that satisfies all equations (Fig. 1). The left side of Fig. 1 shows the main stream procedure. The motion, heat and species modules call the same solver subprogram, detailed in the right side.

The major drawback with this assumption is that the fluid model is unable to predict the shape of the dead man, with the result that the dead man shape must initially be prescribed, leading to special complicated mesh structures (see left side of Fig. 3). Other attempts or improvements tend to introduce the shape during calculation by removing a set of elements from the calculation domain. ${ }^{15)}$ But in this trial, the solid phase is still described by a viscous constitutive equation and is assumed not to enter inside the dead man.

\subsection{Finite Element Approach of Solid Flow}

In the previous section, we have introduced the general transport Eq. (2), described in an Eulerian frame. This means that the arbitrary control volume is fixed in space, and the material flowing through it is described by any field $\Phi$, which depends only on its fixed position in space.

In this section, we shall emphasize the finite element method to solve the solid's phase momentum equation, instead of using the finite volume method described earlier.
This choice is justified by the fact that a better theory for granular materials is now available, but requires the use of FEM.

The Lagrangian description is adopted: the control volume is attached to the material, and is deforming with it. The Lagrangian approach has the advantage to suppress convective terms from equilibrium equations, thus it minimizes the use of spatial derivatives. Most often, Lagrangian problems are associated with transient phenomena, but it is possible to reach steady flow conditions using iterative remeshings.

Computing a steady solid velocity field within the solid flow model indeed requires several remeshings. The remeshing procedure consists of removing elements in the raceway, adding new layers at the top, and transferring nodal and element data from the deformed mesh to the undeformed mesh. This procedure allows a Lagrangian problem to be transformed into an Eulerian problem. During this procedure, the solid velocity field is calculated, as well as the dead man shape (see its definition in Sec. 3).

The general momentum equation in its Lagrangian form is:

$$
\rho \vec{a}+\dot{s} \vec{v}=\vec{\nabla} \cdot \overline{\bar{\sigma}}+\vec{f}
$$

where $\vec{a}=d^{2} \vec{u} / d t^{2}$ is the material acceleration, $\vec{v}=d \vec{u} / d t$ is the velocity field vector, $\rho$ is the material density, $\dot{s}$ the volumetric rate of material source and sink, $\overline{\bar{\sigma}}$ is the true or Cauchy stress tensor field, and $\vec{f}$ is the external body force which gathers gravity and drag forces exerted by other phases.

Note that this equation differs from usual representations of momentum equations by the fact that the material source-sink term $\dot{s}$ appears, and is usually not equal to zero for reacting beds.

The left side of Eq. (3) represents the time variation of material impulse, and the right side represents the sum of all forces exerted on the control volume. These forces include body forces (through $\vec{f}$ ) and surface forces (through the stress tensor).

By making use of the theorem of virtual works, the spatial derivative exerted on the stress tensor is transfered to the virtual strain rate:

$$
\int[\delta \overline{\bar{\varepsilon}}]^{T}: \overline{\bar{\sigma}} d \mathscr{V}=\int(\delta \vec{u})^{T} \cdot\left(\vec{f}-\rho \frac{d^{2} \vec{u}}{d t^{2}}-\dot{s} \frac{d \vec{u}}{d t}\right) d \mathscr{V}
$$

The physical meaning of Eq. (4) is that the virtual work of external forces (scalar product of a virtual displacement increment field by the d'Alembert forces field) is equal to the virtual work of internal forces (double scalar product of a virtual strain rate field, deduced from the previous virtual displacement increment field, by the internal forces field represented by the stress tensor field).

Note that Eq. (3) was an equality between vector fields, whereas Eq. (4) is an equality between scalar fields. Equation (4) is also called the weak form of the momentum equation, because the stress field does not require derivability anymore.

The principle of $\mathrm{FEM}^{16)}$ stipulates that the calculation domain is an assembly of a finite number of cells, called elements, inside which the fields are supposed to vary contin- 
uously. The continuous virtual fields in Eq. (4) are approximated over a finite set of nodes, by means of interpolation functions (function of material coordinates).

The improvement of FEM over FVM is, in fact, that the spatial derivatives used to calculate strain rates are reported directly to interpolation functions, not on nodal values. The variations of the continuous fields are then approximated by the variations at a finite set of nodes. This provides a more rigorous approach than substituting finite differences to partial derivatives at the same nodes (method used in the FVM).

We now define $\mathbf{N}$ the interpolation function, and $\mathbf{B}=$ $\frac{1}{2}\left[(\vec{\nabla} \mathbf{N})+(\vec{\nabla} \mathbf{N})^{T}\right]$ the symmetric part of its spatial derivative, so that:

$$
\begin{aligned}
& d \vec{u}=\mathbf{N} \Delta \mathbf{U} \\
& d \overline{\bar{\varepsilon}}=\mathbf{B} \Delta \mathbf{U}
\end{aligned}
$$

where $\mathbf{U}$ is the array of all nodal displacement degrees of freedom, and $\Delta \mathbf{U}$ its increment.

Therefore, the weak form of the momentum Eq. (4) is written again as:

$$
\int \mathbf{B}^{T}: \overline{\bar{\sigma}} d \mathscr{V}=\int \mathbf{N}^{T} \cdot\left(\vec{f}-\rho \frac{d^{2} \vec{u}}{d t^{2}}-\dot{s} \frac{d \vec{u}}{d t}\right) d \mathscr{V}
$$

The left side of Eq. (7) is the array of nodal internal forces, and the right side is the array of nodal external forces. Their equality expresses the equilibrium of all nodes. Note that Eq. (7) is a system of non-linear equations, whose number is equal to the total number of degrees of freedom.

Since Eq. (7) is non-linear, it is solved incrementally. In order to reach a solution, the granular material constitutive equation is used ${ }^{12)}$ :

$$
d \overline{\bar{\sigma}}=\mathbf{H}: d \overline{\bar{\varepsilon}} .
$$

where $\mathbf{H}$ is the Jacobian of the stress-strain incremental constitutive equation (see Appendix A for more details). Each component of $\mathbf{H}$ represents an anisotropic bulk modulus of the material. The Jacobian is in most general cases a function of stress state, through the tensor $\overline{\bar{\sigma}}$, and of strain increment.

An incremental constitutive law is a relation between strain increment and stress increment. During deformation history, a specific strain path is prescribed to each element. The response in stress is given by integrating Eq. (8) step by step, until the final stress obtained is the one that equilibrates Eq. (7).

We introduce the following arrays:

$$
\mathbf{K}=\int \mathbf{B}^{T}: \mathbf{H}: \mathbf{B} d \mathscr{V}
$$

is the stiffness matrix (it includes the constitutive granular behavior),

$$
\mathbf{C}=\int \mathbf{N}^{T} \cdot \mathbf{N} \dot{s} d \mathscr{V}
$$

is the damping matrix (it includes material source and sink terms),

$$
\mathbf{M}=\int \mathbf{N}^{T} \cdot \mathbf{N} \rho d \mathscr{V}
$$

is the mass matrix (it includes the inhomogeneous density field), and:

$$
\mathbf{R}=\int \mathbf{N}^{T} \cdot \vec{f} d \mathscr{V}-\int \mathbf{B}^{T}: \overline{\bar{\sigma}} d \mathscr{V}
$$

is the residual vector (difference between external forces array and internal forces array).

Therefore, the non-linear set of equations are approximated by the following incremental form:

$$
\mathbf{M} \frac{d^{2}}{d t^{2}} \Delta \mathbf{U}+\mathbf{C} \frac{d}{d t} \Delta \mathbf{U}+\mathbf{K} \Delta \mathbf{U}=\mathbf{R}
$$

and it is usually solved by using Newton based techniques.

After some iterations, the solution provides the incremental nodal displacement field $\Delta \mathbf{U}$ (it includes both the horizontal and vertical displacement degrees of freedom of each node. Note that they were solved separately in the fluid flow model.

Convergence is reached when either the components of the residual array $\mathbf{R}$ or the increment displacement array $\Delta \mathbf{U}$ are sufficiently small. When convergence is reached, the resulting stress field equilibrates the external forces field (the sum of gravity and drag forces exerted by other phases). This equilibrium is accompanied by a general displacement field, which also includes mesh distortion. Mesh distortion is the side effect of the Lagrangian approach: remeshing is necessary in order to reach steady flow conditions. During the remeshing procedure, the velocity field is computed, then exported to the fluid flow model. All these steps are summarized in Fig. 2: the momentum equations are incrementally solved, then the Eulerian velocity field is calculated after remeshing was performed.

Note that the system (13) is very similar to the equation of motion of a mass attached to a spring and a dash-pot:

$$
m \ddot{u}+c \dot{u}+k u=r .
$$

Indeed, the system (13) can be seen as the general form of Eq. (14) in multiple dimensions and a much higher number of nodes. If any perturbation occurs, through external load or boundary conditions, the global system smoothly relaxes until some equilibrium is reached again.

In our case, possible perturbations are change of boundary conditions, e.g. raceway coke combustion, or change of body loads, e.g. density change through cohesive zone that affects gravity load.

\subsection{Coupling Techniques}

The major difficulty was to connect two separate and very different models. Indeed, the numerical methods used to solve transport equations for fluids or momentum equations for solids, are far removed from one another, and the corresponding programs cannot be merged into one global program.

The first choice was to create one unique mesh for both codes (see right side of Fig. 3), in order to treat variables at same nodal or cell geometrical positions. This solution 


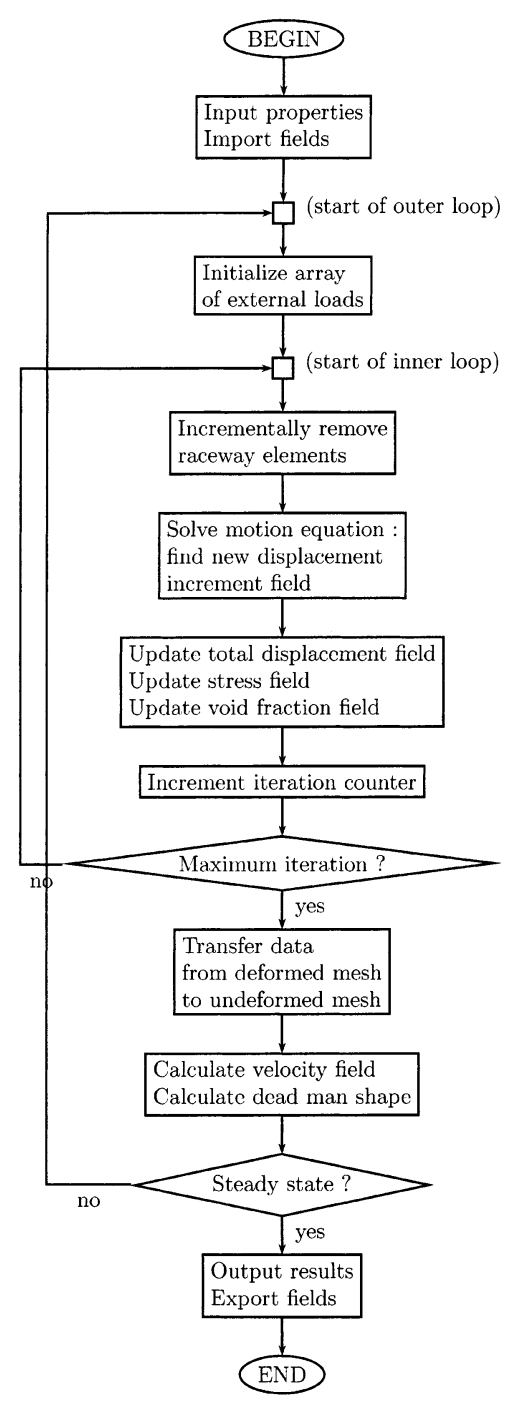

Fig. 2. Solid flow model's algorithm.

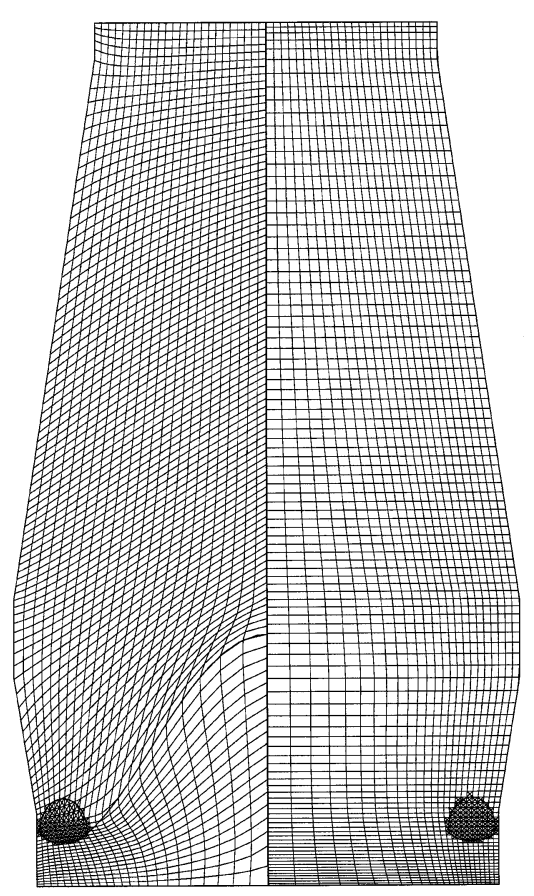

Fig. 3. Comparison between old mesh (left) and new proposed mesh (right) used by both solid flow model and fluid flow model.

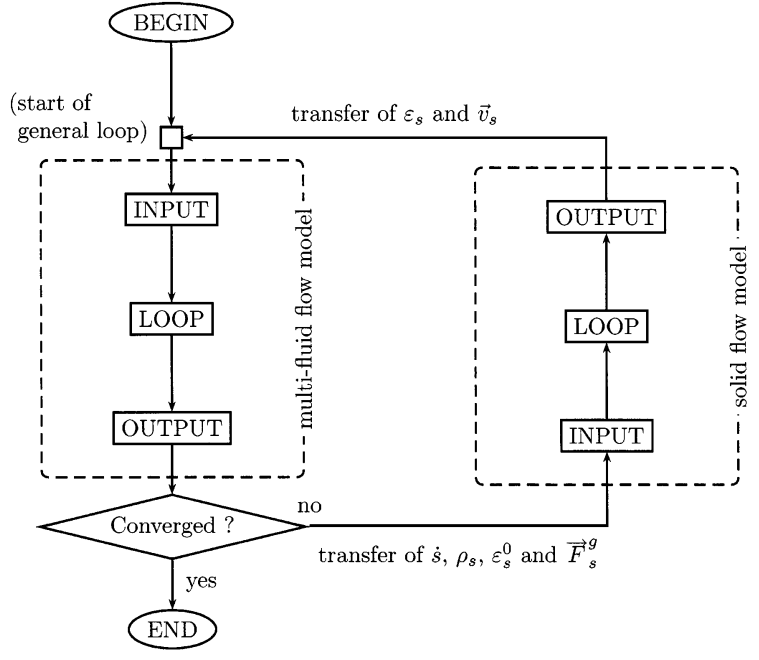

Fig. 4. Multi phases-multi flows coupled algorigthm.

avoids using complicated interpolation and extrapolation procedures. Note that the raceway is at present still prescribed by a simple geometric curve, in which its height and depth are given. The second choice was to run each program one after the other and allow them to communicate by exchanging several kinds of data. This strategy is described in more details in the following paragraphs.

Each model needs a specific set of input data. Basic data needed by the fluid flow model are the following:

1. geometry of blast furnace

2. material characteristics (physical and chemical)

3. operating conditions (like wall heat loss, blast temperature and composition, layers structure, radial distribution of voidage and diameters for both ore and coke layers is implemented in the fluid model).

On the other hand, basic data needed by the solid flow model are:

1. geometry of blast furnace and boundary conditions (like wall angle of friction)

2. solid characteristics (density, voidage)

3. external loads (gravity and interphase drag forces)

4. solid phase source and sink term

Some of the basic input data needed by one model can be prepared by the other model. For example, the following data required by the solid flow model are in fact computed by the fluid flow model (see Fig. 4):

1. Material source and sink rate $\dot{s}$, to ensure continuity.

2. Density field $\rho$ for each cell, to provide gravity load.

3. Drag forces, to simulate interphase interactions. In the case of solid-gas interaction, the anisotropic force $\vec{F}_{\mathrm{s}}^{g}$ depends on the layer's angle $\theta$ (see Appendix B).

4. Voidage field, to provide an initial value for $e_{0}$ in the stiffness equation. The voidage is then updated, by taking into account the solid pressure, before being sent back to the fluid model (see Appendix C).

Based on this information, the solid flow model calculates the solid phase velocity field, and furnishes an updated solid voidage field. These two fields are transfered back to the fluid flow model (Fig. 4). The solid flow model is just an external solver for the solid momentum equations. All other calculations are performed within the multi-fluid model. 
In the multi-fluid flow model, the resolution of solid phase momentum equations is skipped. The required information on the solid phase velocity field is stored prior to calculations. These data are updated less frequently than the other fields. This choice is justified by the difference in range between the solid velocities and the velocities of the other phases.

During the very first step, when the solid velocity field is missing, the multi-fluid model considers only the gas phase flowing in a static un-reactive bed, with some fixed voidage. Therefore, gas-solid drag forces are initialized. The solid flow model is run afterwards in order to compute an initial stress field in a static bed that equilibrates gravity, which depends on an inhomogeneous density field computed by the multi-fluid model, and the aforementioned gas-solid drag force. This procedure updates the solid voidage, and the multi-fluid flow model is run again in order to compute a better approximation of gas-solid drag force.

After this initialization cycle, the solid flow model is run with the updated drag force in dynamic conditions: combustion in the raceway is at first considered alone, which computes a first estimation of the solid phase velocity field. Once this field becomes available, the multi-fluid flow model is run again, this time with all phases being reactive, which computes a first estimation of the solid material source-sink term. After this second cycle, all fields required by both models become available. Therefore, the general procedure continues iteratively and all fields are gradually updated, until convergence occurs.

The gas flow has, at least, three different impacts on solid flow: the first one is on solid temperature, through heat exchanges. The second one is on solid physical properties, like local composition, through chemical reactions. The third one is on the additional force exerted on the burden, through Ergun's equation.

The solid temperature field affects the solid physical properties, including the solid density (mixture of coke and ore above $\mathrm{CZ}$, only coke below). The density is used to calculate the effect of gravity, part of the total external force in the solid flow model. This external force, as well as the gas-solid drag force, are equilibrated by the stress field, considered as an internal force inside the solid flow model. Reaching this new stress field updates the velocity field. Indeed, the hypo-plastic constitutive law is a relation between stress increment and strain increment, (see Eq. (8)), and strain increment is related to velocity.

By inverting the general nodal Eq. (7), a new velocity field is obtained, which takes into account all changes in physical properties, through the mass matrix $\mathbf{M}$ which reflects changes in the density, through the damping matrix $\mathbf{C}$ which reflects changes in solid composition, through the constitutive Jacobian matrix $\mathbf{K}$ which reflects changes in burden's void fraction distribution, through the external forces $\vec{F}_{\text {e }}$ which gathers the influence of gravity and the interaction with other flowing phases.

The minimum number of cycles required depends mainly on the stability of the solid velocity field, which is due to the remeshing procedure. ${ }^{17)}$ Indeed, remeshing is performed after each run through the solid flow model, when solid velocity field is computed. This process is the slowest to stabilize. Since the main input of the fluid flow model is the
Table 2. Blast furnace dimensions in $\mathrm{m}$.

\begin{tabular}{ccccccc}
\hline $\begin{array}{c}\text { throat } \\
\text { radius }\end{array}$ & $\begin{array}{c}\text { belly } \\
\text { radius }\end{array}$ & $\begin{array}{c}\text { hearth } \\
\text { radius }\end{array}$ & $\begin{array}{c}\text { shaft } \\
\text { height }\end{array}$ & $\begin{array}{c}\text { belly } \\
\text { height }\end{array}$ & $\begin{array}{c}\text { bosh } \\
\text { height }\end{array}$ & $\begin{array}{c}\text { hearth } \\
\text { height }\end{array}$ \\
\hline 5.5 & 8.1 & 7.4 & 17.2 & 2.5 & 4.3 & 2.3 \\
\hline
\end{tabular}

Table 3. Material characteristics.

\begin{tabular}{cccccc}
\hline$\rho\left(k g \cdot m^{-3}\right)$ & $\Phi\left(^{\circ}\right)$ & $h_{s}(M P a)$ & $n(-)$ & $e_{0}(-)$ & $\Phi_{w}\left(^{\circ}\right)$ \\
\hline 500 & 35 & 7.0 & 1.1 & 1.0 & 10 \\
\hline
\end{tabular}

solid velocity field, it is considered that once the solid velocity field becomes stabilized then the fields calculated by the fluid model become stabilized as well.

A general criterion based on the solid velocity field is chosen to examine convergence. The solid velocity profile on the central axis has previously been examined for the solid flow model alone, ${ }^{17)}$ and it was found that a minimum of five remeshings were required to obtain a steady velocity field. The same number of iterations is performed within the current study, also to take into account computation time and storage space.

\section{Numerical Simulations Using Coupled Models}

In this section, some results of the coupled models will be introduced. The blast furnace dimensions are summarized in Table 2. The solid phase material parameters, used within the solid flow model, are gathered in Table 3. The blast furnace operating conditions, used within the fluid flow model, can be found elsewhere. ${ }^{10,11)}$

The fields described here concern mainly solid flow inside the blast furnace, but differ from previous publications ${ }^{17)}$ in the fact that they are computed by taking into account the mechanical effect of other flowing phases, like gas, physical transformations, like ore softening and melting, as well as chemical reactions affecting both coke and ore layers with material source and sink terms.

\subsection{Description of Stress Field Inside Solid Phase}

Figure 5 shows the stress field inside the burden. The obtained stress field equilibrates the external forces, like gravity and gas drag forces.

The stress tensor at a point is represented by a cross whose arm lengths are proportional to the principal stress values, and arm directions are oriented according to the eigen-directions of the stress tensors. The orientation of principal stresses are determined mainly by flow conditions under external forces, but also by wall profile and roughness.

The upper part of the blast furnace is in an active state, because the major pressure direction is vertical. This phenomenon is attributed to the additive contributions of a vertical gravity vector and a vertically oriented gas flow.

However, the lower part of the blast furnace is in a passive state, because the major pressure direction is rather horizontal near wall. This is due to the converging flow conditions towards the raceway. Note that an arch of major pressure directions spans radially around the raceway. The extremity of the arch that lies on the belly wall contributes to wall mechanical erosion in this part of the blast furnace. Note that another contribution to wall erosion is hot flowing 


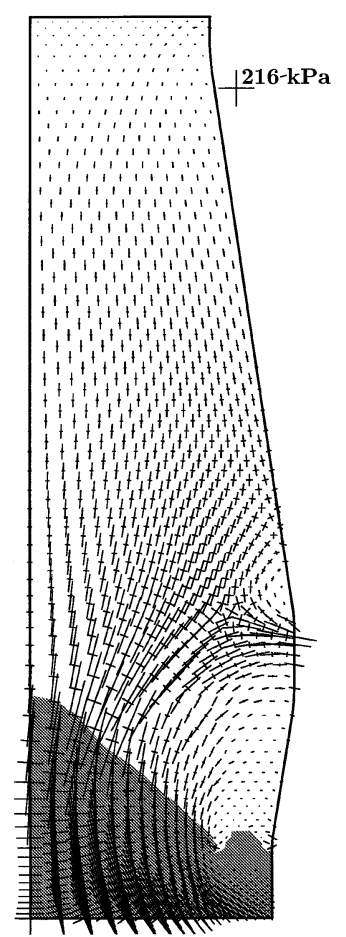

Fig. 5. Stress field in the blast furnace.

gas.

The switch from active stress state to passive stress state occurs at the junction between shaft and belly. In this region, the stress state is rather hydrostatic, since the cross arms have approximatively the same lengths. The region where the switch occurs is crossed by the arch of major pressures.

The major pressure direction remains vertical along the axis, which is coherent with symmetry. The major pressure value increases with depth, but reaches much higher values inside the dead man. This is due to the stronger decrease of velocity in the dead man, which generates compressive strain rate and therefore increases the solid pressure. Note that compressive strain comes from the vertical gradient of vertical velocity which becomes negative.

On the other hand, pressures above the raceway are much lower. This is due to the stronger increase of velocity in this region: the vertical gradient of vertical velocity is positive, thus generating extensive strain rate, and therefore a decrease of solid pressure.

Higher grain compression and friction contributes to granular degradation, which is responsible for fine generation. Solid friction occurs where the velocity modulus varies on a short distance, in other words in high shear regions. For example, horizontal variation of vertical velocity produces shear strain rate, hence shear stress. High shear stress regions are noticeable where the principal directions of the stress tensor are rotating, that is in the arch of major pressures at belly level and at the bottom of the shaft. Gas flow paths cross the regions where fines are most likely to be produced, carrying them away.

\subsection{Definition of Dead Man Boundary}

Figure 6 represents the velocity field inside the solid phase, by means of iso-velocity curves. Their values are a fraction of the maximum velocity, just above the raceway.

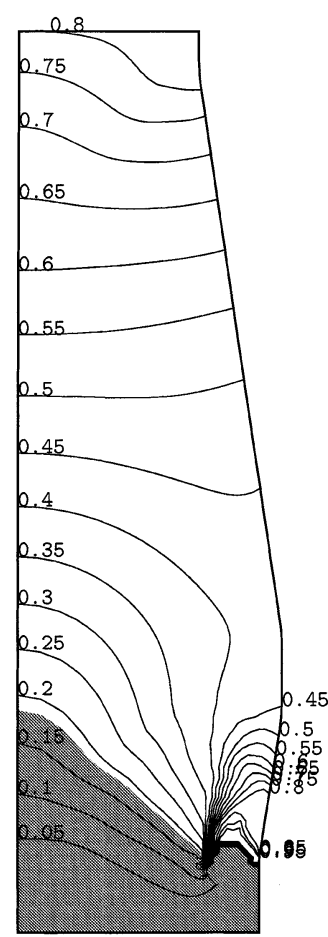

Fig. 6. Iso-velocity curves.

The average top velocity is about $3 \times 10^{-3} \mathrm{~m} \cdot \mathrm{s}^{-1}$.

The iso-velocity curves show clearly a quasi-stagnant region (shaded), bounded by a slow moving zone, and above these two regions a fast moving zone. The quasi-stagnant region belongs to the calculated dead man. Since the velocity is not perfectly equal to zero inside the dead man, its profile should be determined by an iso-velocity curve, the value of which should be some percentage of the characteristic velocity. We therefore need to define the iso-velocity value that bounds the dead-man.

The starting point of this curve on the central axis is the location where the inflexion point of the velocity profile along the central axis occurs. Indeed, the velocity along the central axis is a monotonously decreasing function of height. The inflexion point is characterized by the maximum decrease of velocity (see Fig. 8). We then follow this property through the burden until the other side is reached. We define the resulting curve as the boundary of the dead man. The dead man boundary is the curve where the velocity decrease is maximum. It is interesting to note that the dead man height does not depend on the iteration step number.

\subsection{Description of Solid Velocity Field}

Figure 7 is another representation of the velocity field, by means of trajectories and time lines. Markers were placed where time lines intersect trajectories. Along a trajectory, each marker is spaced by $10 \mathrm{~min}$. Intentionally, the top time line does not coincide with the top mesh line in order to follow the evolution with depth of the slope of the burden layers. Note that the mesh top line has to be kept horizontal to help the remeshing procedure.

A plug flow can be seen in the upper part, where the trajectories are equally spaced, and a funnel flow can be seen in the lower part, where the trajectories are converging towards the raceway. These two properties are related to the 


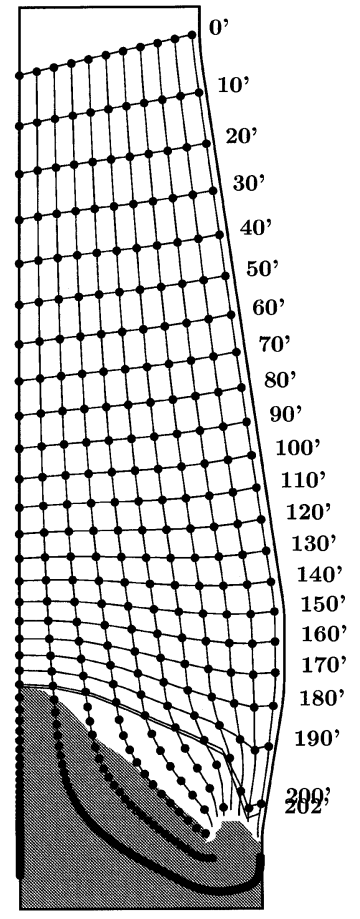

Fig. 7. Trajectories and time lines.

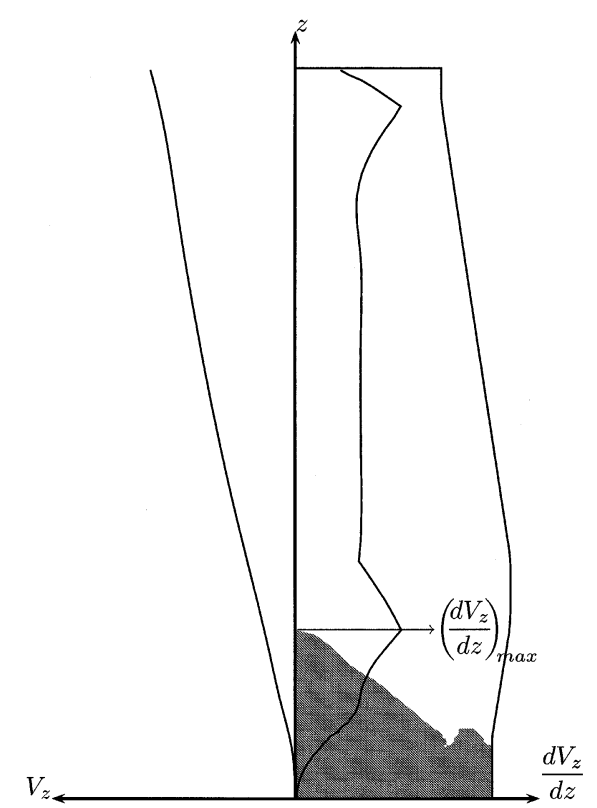

Fig. 8. Velocity derivative profile on central axis.

active state and passive state fields that describe the stress field.

In the plug flow region, iso-velocity curves are rather horizontal. In this region, the shear level is rather low, hence fines are not generated. On the other hand, in the funnel flow region, all iso-velocity curves end at the raceway boundary, becoming vertical. A higher horizontal gradient of vertical velocity is responsible for shear, and therefore for fine generation.

Time lines inclination indicates the evolution of the layer's angle with time. The angle of time lines is used in the fluid model to compute anisotropic Ergun's forces $\vec{F}_{\mathrm{s}}^{g}$. It can be seen that the ore/coke layers become more and more flat with depth. This is due to a conjugated effect of the raceway, that attracts the layers on the wall side, and of the dead man, that slows down the layers descending motion on the axis side. The space between the time lines becomes also more and more narrow in the shaft and belly: this is due to the increase of radius and of occupied surface. The occupied volume remains the same, hence the decrease of thickness.

The dead man region is characterized by very slow motion. To assert this point, it can be seen in Fig. 7 that the space between the markers along the trajectories is very tight once the dead man was penetrated, whereas the space is much looser above dead man. The calculated residence time inside the dead man is more or less equal to $25 \mathrm{~h}$. Note that the dead man tends also to be consumed by the raceway's side and bottom: this fact was also observed during small scale two dimensional and three dimensional experiments. $^{18)}$

\section{Conclusion}

The sophistication of an existing blast furnace total model is increased by connecting it to an external solid flow model for granular materials based on hypo-plasticity theory. The former model is based on the finite volume technique, and the latter model is based on the finite element technique. Each program is run one after the other and exchange data like volume forces and material source and sink terms on one hand, and solid velocity field and an update of voidage on the other hand. The solid momentum equations are no longer within the multi-fluid flow model.

The purpose of the incremental finite element method is to reach the limit state, where steady state flow occurs. To reach this state, starting from a state at rest, an evolution equation is used, which relates stress increments to strain increments. This equation is incrementally integrated with the FEM.

The solid flow model furnishes a better understanding of mechanical phenomena that affects the burden, and is able to predict the shape of the dead man by means of an iso-velocity curve. The starting point on the central axis of this curve is by definition the location of the inflexion point for velocities (where they decrease the most). The dead man is not static: residence time inside the dead man was numerically found to be $25 \mathrm{~h}$ in some points. Therefore, the dead man is not a boundary for solid flow.

By examining both solid stress field and solid velocity field, different characteristic regions in the blast furnace were sketched out, like active and passive state regions for the stress field, or plug and funnel flow regions for the velocity field. Solid velocities were found to be higher where solid pressures were found to be lower, and conversely, solid velocities were found to be lower where solid pressures were found to be higher.

The switch between these regions is an arch of major pressures that was found to span from the dead man surface until the belly wall. This arch is a location of high shear, and is therefore, the region where fine generation may occur. This phenomenon should be taken into account in future research developments of coupled models.

A minimum amount of five iterations between the two 
models is required before reaching a steady state solid flow, which then enables steady state fields to develop within the multi-fluid model. This numerical coupling between two different codes brought more reliable results than the multifluid flow model alone with a solid phase assumed to behave like a fluid.

These arguments demonstrate that the methodology proposed here is the most reasonable for simulating blast furnace phenomena at the present. Further results obtained with the coupled models, like solid temperature, ore reduction curves, or gas stream lines, will be presented in future publications.

\section{Nomenclature}

$$
\begin{array}{ll}
\rho: & \operatorname{density}\left(\mathrm{kg} \cdot \mathrm{m}^{-3}\right) \\
\mu: & \text { viscosity }(\mathrm{Pa} \cdot \mathrm{s}) \\
\phi: & \text { granular internal angle of friction }\left(^{\circ}\right)
\end{array}
$$

$e^{0}, e:$ granular void ratio at rest and in dynamic conditions (-)

$\varepsilon_{m}^{0}, \varepsilon_{m}$ : granular voidage ratio at rest and in dynamic conditions (-)

$P: \quad$ solid pressure $(\mathrm{Pa})$

$\dot{s}: \quad$ material source-sink term $\left(\mathrm{kg} \cdot \mathrm{m}^{-3} \cdot \mathrm{s}^{-1}\right)$

$\vec{u}$ : displacement $(\mathrm{m})$

$\vec{v}:$ velocity $\left(\mathrm{m} \cdot \mathrm{s}^{-1}\right)$

$\vec{a}$ : acceleration $\left(\mathrm{m} \cdot \mathrm{s}^{-2}\right)$

$\vec{f}$ : external volumetric forces $\left(\mathrm{N} \cdot \mathrm{m}^{-3}\right)$

$\overline{\bar{\sigma}}: \quad$ stress tensor $(\mathrm{Pa})$

$\overline{\bar{\varepsilon}}: \quad$ strain tensor $(-)$

H : constitutive equation's Jacobian $(\mathrm{Pa})$

\section{REFERENCES}

1) Y. Omori: Blast furnace phenomena and modelling. Elsevier Applied Science, London, (1987), 1.

2) J. Yagi: ISIJ Int., 33 (1993), 619-639.

3) T. Sugiyama and M. Sugata: Seitetsu Kenkyu, 325 (1987), 34-43.

4) T. Sugiyama and I. Yamaguchi: CAMP-ISIJ, 9 (1996), 18-21.

5) K. Takatani, T. Inada and Y. Ujisawa: CAMP-ISIJ, 7 (1994), 50-53.

6) K. Takatani: Tetsu-to-Hagané, 81 (1995), 1031-1036.

7) T. Inada, K. Takatani, M. Miyahara and H. Yamaoka: ICSTI/ Ironmaking Conf. Proc., ISS, Warrendale PA Vol. 57, (1998), 465-470.

8) M. Hatano and K. Kurita: Tetsu-to-Hagané, 66 (1898), 1980

9) J. Yagi, K. Takeda and Y. Omori: Trans. Iron Steel Inst. Jpn., 22 (1982), 884-892.

10) P. R. Austin, H. Nogami and J. Yagi: ISIJ Int., 37 (1997), 458-467.

11) P. R. Austin, H. Nogami and J. Yagi: ISIJ Int., 37 (1997), 748-755.

12) S.-A. Zaïmi, T. Akiyama J.-B. Guillot and J. Yagi: ISIJ Int., 40 (2000), No. 4, 332-341.

13) J. Chen, T. Akiyama, H. Nogami, J. Yagi and H. Takahashi: ISIJ Int., 33 (1993), 664-671.

14) S. V. Patankar: Numerical Heat Transfer and Fluid Flow, McGrawHill, New York, (1980), 1.

15) S. J. Zhang, A. B. Yu, P. Zulli, B. Wright and U. Tüuzün: ISIJ Int., 38 (1998), 1311-1319.

16) K. J. Bathe: Finite Element Procedures. Prentice Hall, (1996), 1.

17) S.-A. Zaïmi, J.-B. Guillot, Ch. Petit and J.-M. Steiler: ICSTI/ Ironmaking Conf. Proc., ISS, Warrendale PA Vol. 57, (1998), $343-350$.

18) H. Takahashi: CAMP-ISIJ, 12 (1999), 81-84.

19) S. Ergun: Chem. Eng. Prog., 48 (1952), 89-94.

20) I. Herle: in print, Mech. of Coh. Fric. Mat., (1999).

21) T. Yamada, M. Sato, N. Miyazaki, H. Shimamura and S. Taguchi: Kawasaki Steel Technical Report, 6 (1974), 16-37.

22) Y. Ohno, Y. Yamada, K. Kondo and T. Takebe: Tetsu-to-Hagané, 73 (1987), 234-241.
23) H. Yamaoka: Tetsu-to-Hagané, 72 (1986), 2194-2201.

\section{Appendix A. Constitutive Equation}

The granular bed (the burden) is described by a hypoplastic constitutive equation. ${ }^{12)}$ In this theory, the Jacobian $\mathbf{H}$ that appears in Eq. (8) is a function of stress tensor $\overline{\bar{\sigma}}$ and strain increment $d \overline{\bar{\varepsilon}}$ :

$$
\mathbf{H}(\overline{\bar{\sigma}}, d \overline{\bar{\varepsilon}})=f_{\mathrm{s}}\left(\mathbf{L}(\overline{\bar{\sigma}})+\mathbf{N}(\overline{\bar{\sigma}}) \otimes \frac{d \overline{\bar{\varepsilon}}}{\|d \overline{\bar{\varepsilon}}\|}\right) \ldots \ldots . .(\mathrm{A}
$$

where $f_{s}$ is the stiffness coefficient. The characteristics of this constitutive equation follow:

1. Non-linearity: this is introduced by the strain increment direction $d \overline{\bar{\varepsilon}} /\|d \overline{\bar{\varepsilon}}\|$, which provides irreversibility, even for very small cycles. Plasticity appears as soon as deformation occurs. The same function is used to describe loading as well as unloading, whereas two functions are needed in elasto-plasticity.

2. Yield surface: the failure information is defined by

$$
\begin{gathered}
\operatorname{tr}\left(\mathbf{L}^{-1}: \mathbf{N}\right)=0 . \\
\left\|\mathbf{L}^{-1}: \mathbf{N}\right\| \ldots .
\end{gathered}
$$

where the stress increment is equal to zero for non zero strain increments. This phenomenon, typical for granular materials, is called critical or limit state. Note that at critical state, the volumetric strain rate is equal to zero. The previous equations should lead to a relation between stress invariants, called the criterion, which is a function of the internal angle of friction.

3. Rate independence: the Jacobian $\mathbf{H}$ is homogeneous of degree zero towards strain rate: it depends only on its direction, not its modulus. This property allows one to simulate deformations in granular beds at any speed.

\section{Appendix B. External Loads}

In this section we describe the external loads acting on the granular bed. In the fluid flow model, they are responsible for the motion of the solid phase. These external loads appear in the right hand side of the momentum Eq. (2), as source terms. In fact, the source term for solid phase momentum equation consist of pressure gradient and interphase drag force (also called momentum transfer). The interphase drag force include interaction between solid on one side and gas, liquid and fines on the other side.

In the solid flow model, external forces consist of gravity and interphase drag force only. These are responsible for the generation of an internal stress field that equilibrates the external forces. The missing pressure is included in the stress field, in its hydrostatic part. The deviatoric part is related to the yielding of the granular bed. Once yield occurs, the bed flows steadily.

A simplified form of the interphase drag force is used: only solid-gas interaction is considered (also known as pressure drop or Ergun's force ${ }^{19)}$ ), in an anisotropic form to take into account the slope of the burden layers ${ }^{8,23)}$ (but not their thickness). Each solid component (coke, sinter, pellet, scrap) contributes to the resistance to gas flow through par- 
ticle diameter $d_{m}$, voidage $\varepsilon_{m}$ (see below) and particle shape $\varphi_{m}$ :

$$
\begin{aligned}
& F_{m}=\left[1.75 \rho_{\mathrm{g}}\left|\vec{V}_{\mathrm{g}}\right|+150 \mu_{\mathrm{g}}\left(\frac{\varepsilon_{m}}{\left(1-\varepsilon_{m}\right) d_{m} \varphi_{m}}\right)\right] \\
& \times\left(\frac{\varepsilon_{m}}{\left(1-\varepsilon_{m}\right) d_{m} \varphi_{m}}\right)
\end{aligned}
$$

Here, $\vec{V}_{\mathrm{g}}, \rho_{\mathrm{g}}$ and $\mu_{\mathrm{g}}$ are gas velocity, gas density and gas viscosity respectively. Parallel and perpendicular components are computed from averaged values of Eq. (A-4):

$$
\begin{gathered}
F_{\perp}=\sum_{m} f_{m} F_{m} \\
\frac{1}{\sqrt{F_{\|}}}=\sum_{m} \frac{f_{m}}{\sqrt{F_{m}}}
\end{gathered}
$$

where the ponderation coefficients $f_{m}$ are the volumetric fractions of each components within solid phase. Note that:

$$
\sum_{m} f_{m}=1
$$

Finally, the drag force vector is obtained by combining parallel and perpendicular components:

$$
\vec{F}_{\mathrm{s}}^{g}=\left(\begin{array}{cc}
\cos \theta & -\sin \theta \\
\sin \theta & \cos \theta
\end{array}\right) \cdot\left(\begin{array}{cc}
F_{\perp} & 0 \\
0 & F_{\|}
\end{array}\right) \cdot\left(\begin{array}{cc}
\cos \theta & \sin \theta \\
-\sin \theta & \cos \theta
\end{array}\right) \cdot \vec{V}_{\mathrm{g}}
$$

where $\theta$ is the local layer's angle.

The gravity force is proportional to the overall solid density field, equal to the average value of the densities of all solid components:

$$
\rho_{\mathrm{s}}=\sum_{m} f_{m} \rho_{m}
$$

\section{Appendix C. Pressure Dependence on Voidage}

The derivation of solid-gas drag force depends on the voidage of each phase, as in Eq. (A-4). The voidage itself depends on grain diameter ${ }^{21)}$ according to:

$$
\varepsilon_{m}^{0}=1-\left[0.153 \log _{10}\left(d_{m}\right)+0.724\right]
$$

for coke material and:

$$
\varepsilon_{m}^{0}=1-\left[0.403\left(100 d_{m}\right)^{0.14}\right.
$$

for ore material.

The grain size varies along the radius of the blast furnace (except inside the dead man, where its value is assumed to be constant), but remains the same during calculations: there is not yet any feed-back to take into account the influence of chemical reactions or mechanical degradation on grain size. Nevertheless, in this way, gas path is strongly dependent on grain size distribution.

It was recently demonstrated ${ }^{20)}$ that the void ratio (ratio of empty volume over filled volume) inside granular beds at rest is a function of solid mean pressure $P$ (the hydrostatic part of the stress tensor):

$$
e=e^{0} \exp \left[-\left(\frac{3 P}{h_{\mathrm{s}}}\right)^{n}\right]
$$

The coefficient $h_{\mathrm{s}}$, called granulate hardness, and the exponent $\mathrm{n}$ are related to the shape of the grain. This model is part of the hypo-plasticity theory.

The void ratio at zero pressure $e^{0}$ is related to the voidage $\varepsilon_{m}^{0}$ (ratio of filled volume over total volume) by the following couple of formulas:

$$
\begin{gathered}
e^{0}=\frac{1}{\varepsilon_{m}^{0}}-1 \\
\varepsilon_{m}=\frac{1}{1+e}
\end{gathered}
$$

Therefore, the pressure dependent voidage is:

$$
\varepsilon_{m}=\frac{1}{1+\left(\frac{1}{\varepsilon_{m}^{0}}-1\right) f(P)}
$$

where:

$$
f(P)=\exp \left[-\left(\frac{-\operatorname{tr}(\overline{\bar{\sigma}})}{h_{\mathrm{s}}}\right)^{n}\right]
$$

is the pressure coefficient, which depends on the stress field, and $\varepsilon_{m}^{0}$ is given by either Eq. (A-10) or (A-11). 